\title{
Bouncing of polymeric droplets on liquid interfaces
}

\author{
S. Gier, ${ }^{1}$ S. Dorbolo, ${ }^{2}$ D. Terwagne, ${ }^{2}$ N. Vandewalle, ${ }^{2,1}$ and C. Wagner ${ }^{1, *}$ \\ ${ }^{1}$ Experimentalphysik, Universität des Saarlandes, 66123 Saarbrücken, Germany \\ ${ }^{2}$ GRASP, Physics Department B5, Université de Liège, B-4000 Liège, Belgium
}

(Received 25 January 2012; published 17 December 2012)

\begin{abstract}
The effect of polymers on the bouncing behavior of droplets in a highly viscous, vertically shaken silicone oil bath was investigated in this study. Droplets of a sample liquid were carefully placed on a vibrating bath that was maintained well below the threshold of Faraday waves. The bouncing threshold of the plate acceleration depended on the acceleration frequency. For pure water droplets and droplets of aqueous polymer solutions, a minimum acceleration amplitude was observed in the acceleration threshold curves as a function of frequency. The bouncing acceleration amplitude for a droplet of a dilute aqueous polymer solution was higher than the acceleration amplitude for a pure water droplet. Measurements of the center of mass trajectory and the droplet deformations showed that the controlling parameter in the bouncing process was the oscillating elongational rate of the droplet. This parameter can be directly related to the elongational viscosity of the polymeric samples. The large elongational viscosity of the polymer solution droplets suppressed large droplet deformations, resulting in less chaotic bouncing.
\end{abstract}

DOI: 10.1103/PhysRevE.86.066314

PACS number(s): 47.50.Gj, 47.35.Pq, 47.55.dk

\section{INTRODUCTION}

In this paper, we studied the effect of droplets of an aqueous polymer solution on a vibrating, highly viscous silicone oil bath. We show that the deformation mode of the droplets plays a crucial role in the dynamics of the bouncing. The non-Newtonian properties of the fluid were found to affect droplet bouncing in terms of the vibration-induced delayed coalescence.

In nature, liquid droplets commonly bounce on solid or liquid interfaces. Raindrops can fall and bounce on soil, plant leaves, or water surfaces. Bouncing is also an important phenomenon in many industrial processes, such as spray painting, pesticide deposition, or inkjet printing. The impact of a simple liquid droplet can produce notably rich and complex dynamics, including the occurrence (or the absence) of splashing [1] or rebounding [2] or the formation of a corona [3]. The physical properties of the liquid droplet, such as the viscosity, surface tension, and density, together with the properties of the impacted surface, can significantly affect bouncing dynamics. To control bouncing, the effects of hydrophobicity [2], temperature (Leidenfrost effect) [4], and the microtexture [5] of the substrate have been previously studied. Bouncing also depends on whether the surface is stationary or in motion [6].

Recent scientific interest has focused on the non-Newtonian effects of an impacting droplet and the droplet splash. Due to their versatility, polymeric liquids play an important role in technical and industrial applications. The addition of a small amount of polymer can alter the splash behavior of an impacting water droplet to the extent that almost no bouncing occurs [7]. When a high-velocity Newtonian droplet impacts a solid substrate, the drop is strongly deformed and breaks into several smaller droplets. At low impact velocities, the drop adheres to the substrate [2]. A liquid drop with an

\footnotetext{
*c.wagner@mx.uni-saarland.de; http://www.uni-saarland.de/fak7/ wagner/.
}

intermediate velocity can also bounce after impacting the solid substrate. The initially spherical drop spreads similarly to a pancake upon impact with the substrate and later quickly retracts and bounces. The addition of a small amount of flexible polymer to the Newtonian liquid can reduce the rebound of the drop [7]. Polymers do not noticeably change the shear viscosity of a polymeric solution but can strongly affect the elongational viscosity of the solution through normal stresses. Non-Newtonian effects become dominant because the flow inside the drop is elongational. More precisely, the contact line dynamics of the drop is governed by the competition between the surface tension, which favors retraction, and the elastic normal stresses, which slow retraction [8].

When a liquid droplet strikes a liquid surface at rest, the droplet either coalesces immediately or remains on the surface before coalescing. The delay in coalescence is caused by a thin air film between the droplet and the bulk liquid or bath. This film has to drain out for the drop to coalesce with the bath. Newtonian liquid drops can partially coalesce when they are carefully deposited on a liquid surface. Approximately $12 \%$ of a drop merges with the bath whereas the remainder of the drop rebounds: this process can occur several times before the drop coalesces completely $[9,10]$. The effect of a non-Newtonian fluid on coalescence was first discovered by Chen et al. [11]. The authors investigated the coalescence of droplets of polymer solutions on a bath at rest and found that a small amount of polymer in the droplet could completely suppress partial coalescence. Due to the high elongational viscosity of the droplet solution, a connecting filament was always present between the coalesced drop and the residual droplet; this filament prevented further bouncing and immediately led to complete coalescence. These authors also found that the rest time of the droplets was slightly changed at the highest polymer concentration.

Coalescence can be prevented simply by vertically vibrating the bath [12-20] in a sinusoidal motion $A \sin (\omega t)$, where $A$ is the amplitude of the motion and $\omega$ is the angular frequency. In this study, we consider a bath of a highly viscous silicone oil. Consequently, the bath deformation can 
be neglected relative to the droplet deformation. The air film between the droplet and the bath can be renewed under certain conditions (which are discussed below). The squeezed air film generates a vertical lubrication force that prevents coalescence. However, coalescence is prevented only if the acceleration amplitude $A \omega^{2}=A(2 \pi f)^{2}$ is above a threshold value. The threshold $\Gamma_{b}$ depends on the driving frequency of the shaker $f$, the droplet size, and the physical properties of the liquid. The normalized critical acceleration amplitude $\tilde{\Gamma}_{b}=A \omega^{2} / g$ (where $g$ is gravity) for bouncing depends on the characteristics of both the droplet and the bath. In Ref. [12], both the droplet and the bath consisted of viscous silicone oil such that the threshold varied monotonically with the frequency. In general, as reported in Ref. [15], a low-viscosity drop bounces on a high-viscosity bath, and the deformation (or at least the bath dynamics) is negligible due to the viscosity contrast. This configuration produces a resonance process and a minimum acceleration threshold below the acceleration of gravity. On the contrary, for a highly viscous droplet, the droplet deformations becomes negligible, and the minimum in $\Gamma_{b}$ disappears.

The bouncing of polymer solution droplets is related to the problem of a low-viscosity droplet bouncing on a highviscosity bath. The effect of the non-Newtonian liquid on the bouncing of the droplet is as follows [15,20]: the droplet deformation is the predominant mechanism for the bouncing process for droplet diameters that are larger than $1 \mathrm{~mm}$. The aim of the present paper is to study the bouncing of droplets of an aqueous polymer solution on a high-viscosity silicone oil bath. The stresses induced by the deformation from each bounce influence the bouncing behavior. Our study focuses on bouncing (millimeter-sized) droplets on a vibrating surface. We will show that polymers affect the critical threshold for bouncing and qualitatively alter bouncing behavior, even below the overlap concentration $c^{*}$. These effects are related to the high elongational viscosity of a polymeric droplet undergoing an oscillatory elongational deformation $[21,22]$.

\section{EXPERIMENTAL SETUP}

A schematic of the experimental setup is shown in Fig. 1. A flat, round beaker was mounted on top of a shaker, which vibrated the system at a fixed frequency. The beaker was filled to a height of $3 \mathrm{~cm}$ with a silicone oil [Dow Corning Fluid 200,

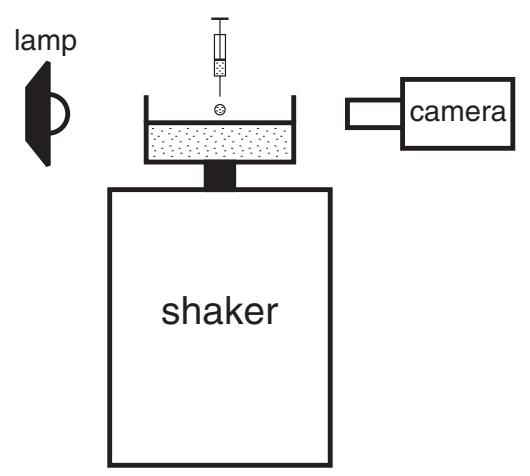

FIG. 1. Experimental setup to investigate the coalescence and bouncing of liquid droplets. kinetic viscosity at room temperature $v=1000$ centistokes (cS)]. The cannula of a syringe that was filled with the sample liquid was placed several millimeters above the liquid surface. The size of the droplet was controlled by varying the diameter of the cannula.

A high-speed camera (MotionPro, IDT, Tallahassee, FL; frame rate, 1000 frames per second; resolution, $640 \times 480$ pixels; shutter speed, $1 / 1000 \mathrm{~s}$ ) was used to record the motion of the droplet. The entire setup was illuminated by a light-emitting diode lamp that was synchronized with the camera. The deformation of the droplet on the bath surface was measured as a function of time. Three main measurements were performed: (i) the bouncing threshold as a function of the frequency, (ii) trajectory analysis of a bouncing droplet, and (iii) droplet deformation during bouncing. The acceleration amplitude thresholds were compared for droplets containing three sample fluids: (i) water, (ii) a surfactant solution (water and Triton X100, at a concentration ten times the critical micelle concentration), and (iii) an aqueous polymer solution [water and $300 \mathrm{ppm}$ polyethyleneoxide (PEO), molecular weight $4 \times 10^{6} \mathrm{amu}$. The effect of surface tension on the bouncing threshold was studied using the water and surfactant solution, while the effect of viscoelasticity on bouncing was studied using the polymer solution.

\section{RHEOLOGICAL PROPERTIES OF THE SAMPLE LIQUIDS}

The liquids used in this study were rheologically characterized in both shear and elongational flows [23]. The shear viscosity was measured using a cone-plate geometry in a commercial rheometer (Haake MARS; cone angle, $2^{\circ}$; cone and the plate diameter, $60 \mathrm{~mm}$ ). The shear viscosities of water and $0.4 \mathrm{mM}$ Triton in water were equal within the experimental resolution ( $\eta=1 \pm 0.05 \mathrm{mPa} \mathrm{s})$, and the shear viscosity of the $300 \mathrm{ppm}$ PEO solution was $\eta=1.3 \pm 0.05 \mathrm{mPa}$ s. The polymer solution did not show any significant shear thinning. Oscillatory shear experiments were conducted, but the elastic modulus could not be determined because the polymer solution was not sufficiently elastic. The overlap concentration $c^{*}=$ $600 \mathrm{ppm}$ of the PEO (molecular weight $4 \times 10^{6} \mathrm{amu}$ ) was determined from the intrinsic viscosity by a dilution series.

High-molecular-weight polymer solutions at low concentrations and correspondingly low viscosity are known to be weakly elastic in shear flow, but the elasticity is strongly altered by elongational flow. Polymeric tumbling in shear flow averages out the stresses. However, the alignment of polymers along the streamlines of an extensional flow effectively stretches the polymers. Large elastic stresses build up until the polymer chains are fully extended, and the relevant material constant is the elongational viscosity $\eta_{e}$. For Newtonian fluids, the elongational viscosity $\eta_{e}$ is three times the shear viscosity: $\eta_{e}=3 \eta$. This relationship is called Trouton's ratio [24]. The transition from the coiled state of the polymer to the elongated state, or the coil-stretch transition [25], occurs only for sufficiently strong flows, i.e., if the elongational rate $\dot{\varepsilon_{c}} \geqslant \frac{1}{2} \lambda^{-1}$, where $\lambda$ is the relaxation time of the polymer. The Weisenberg number is the ratio of the flow rate to the polymer relaxation time, and the critical Weisenberg number is defined by $\mathrm{Wi}_{c}=\dot{\epsilon}_{c} \lambda$. It is nontrivial to determine the elongational 
TABLE I. Summary of intrinsic fluid parameters: density $\rho$, surface tension $\sigma$, dynamic viscosity $\eta$, and the ratio between the viscosity and the surface tension $\eta / \sigma$. The experimental error is mostly due to temperature variations.

\begin{tabular}{lccrr}
\hline \hline Substances & $\begin{array}{c}\rho \\
\left(\mathrm{kg} / \mathrm{m}^{3}\right)\end{array}$ & $\begin{array}{c}\sigma \\
(\mathrm{mN} / \mathrm{m})\end{array}$ & $\begin{array}{c}\eta \\
(\mathrm{mPa} \mathrm{s})\end{array}$ & $\begin{array}{c}\eta / \sigma \\
(\mathrm{s} / \mathrm{m})\end{array}$ \\
\hline Water & $1000 \pm 10$ & $73 \pm 3$ & $1 \pm 0.05$ & 0.014 \\
300 ppm PEO in water & $1000 \pm 10$ & $61 \pm 2$ & $1.3 \pm 0.05$ & 0.021 \\
$0.4 \mathrm{mM}$ Triton in water & $1000 \pm 10$ & $27 \pm 2$ & $1 \pm 0.05$ & 0.037 \\
\hline \hline
\end{tabular}

viscosity of a polymer solution; however, an instrument known as the capillary breakup extensional rheometer (CABER) has recently become available that can be used to determine an apparent elongational viscosity [23,26]. In a CABER experiment, a droplet is placed between two plates that are subsequently separated. The capillary bridge between the plates starts to thin, forming a parallel filament for polymer solutions that thins exponentially in time. For the polymer solutions considered in this study, the flow in this filament is almost purely extensional [27-29]: within the Oldroyd-B model, the characteristic time for the thinning process is simply related to the polymeric relaxation time $\lambda$ by a factor of 3 . For the $300 \mathrm{ppm}$ PEO solution, $\lambda_{c}$ was found to be $20 \mathrm{~ms}$, so that $\lambda \approx$ $6.7 \mathrm{~ms}$. An apparent elongational viscosity $\eta_{e}$ can be deduced from the polymer relaxation time. Elastic stresses continue to grow while the filament is thinning, resulting in an exponential growth of the elongational viscosity. Thus, the elongational viscosity that is determined by the CABER is a time-dependent quantity. The extensional viscosity for the polymer solution at a maximum filament stretch of $\eta_{e} \sim 10^{5} \mathrm{mPa}$ s was found to be almost five decades larger than the elongational viscosity of the solvent. Of course, such large values are only to be expected for a flow rate of at least $\dot{\varepsilon_{c}} \geqslant \frac{1}{2} \lambda^{-1}$ and for sufficiently large values of the so-called Henky strain $\int \dot{\varepsilon} d t$.

Other relevant physical parameters of the liquids, such as the density $\rho$, the surface tension $\sigma$, the dynamic viscosity $\eta$, and the ratio between viscosity and surface tension $(\eta / \sigma)$, are summarized in Table I.

\section{EXPERIMENTAL RESULTS}

\section{A. Bouncing threshold}

A container filled with a highly viscous liquid (Dow Corning Fluid 200, $v=1000 \mathrm{cS}$ ) was vertically vibrated at a fixed frequency. The acceleration amplitude $\Gamma=A \omega^{2}$ was increased stepwise but was always maintained below the threshold of Faraday waves [30]. A droplet of the sample liquid was placed on the surface of the bath. If the droplet bounced for more than $1 \mathrm{~min}$, it was considered to be a bouncer. A 1 min threshold was chosen because droplets either merged within a maximum of a few seconds or were never observed to merge. This process was repeated at least ten times to obtain an adequate statistical distribution. As the acceleration amplitude was increased, new droplets were placed on the surface. The acceleration amplitude beyond which a droplet bounced on the bath surface was defined as the acceleration threshold $\Gamma_{b}$.

The acceleration thresholds for the three different sample liquids are shown in Fig. 2. The threshold was strongly

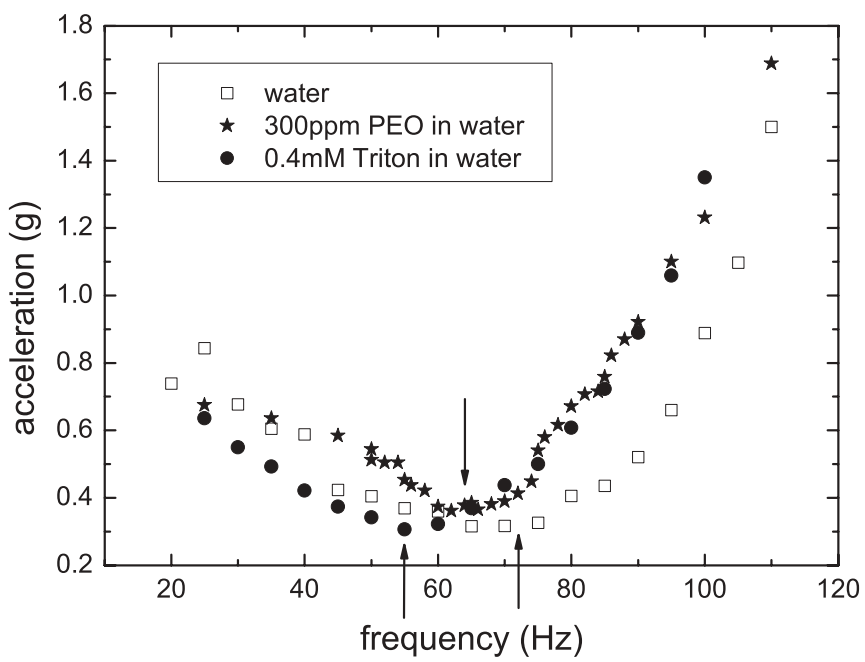

FIG. 2. The acceleration threshold $\Gamma_{b}$ for bouncing droplets consisting of water, $300 \mathrm{ppm}$ PEO in water, and $0.4 \mathrm{mM}$ Triton in water, as a function of the vibration frequency. The arrows indicate the positions of the acceleration minima. For frequencies $f>35 \mathrm{~Hz}$, the statistical error bars are the size of the symbols shown.

frequency dependent for all three samples, and a minimum was observed in the $\Gamma_{b}(f)$ curves. Clearly, the critical acceleration was $\Gamma_{b}<1$ for frequencies up to approximately $f \sim 100 \mathrm{~Hz}$. The largest threshold values corresponded to the polymer solution. Two parameters can be determined from this plot (Fig. 2): the frequency $f_{\text {min }}$ corresponding to the minimum in the $\Gamma_{b}(f)$ curve and the local curvature around the minimum. As shown in Table II, the lowest value of $f_{\text {min }}$ occurs at approximately $55 \mathrm{~Hz}$ for the surfactant solution. The next largest value of the frequency minimum occurs at approximately $65 \mathrm{~Hz}$ for the polymer solution and the largest value occurs at $72 \mathrm{~Hz}$ for water. Non-Newtonian effects were also manifested by a larger local curvature at $f_{\min }$ for the polymer solution than for the other two fluids.

These different observations can be interpreted in the light of the physics of droplet bouncing. The model developed in Ref. [15] is based on (i) applying Newton's second law to the droplet center of mass, accounting for the forcing oscillation and the lubrication force generated by the squeezed film between the droplet and the bath, and (ii) the energy dissipation due to the droplet viscosity and the power developed by the lubrication force. In the frequency range of $0-100 \mathrm{~Hz}$, a normal oscillation mode (described by the spherical harmonic $Y_{2}^{0}$ ) is initiated close to the acceleration threshold (cf. Fig. 5). In Ref. [15] three signature characteristics for low-viscosity liquid droplets bouncing on a viscous bath are discussed:

TABLE II. $\Gamma(f)$ curve minimum and acceleration threshold $\Gamma$ at four different frequencies $(25,50,75$, and $100 \mathrm{~Hz})$.

\begin{tabular}{lcccccc}
\hline \hline & $f_{\min }$ & \multicolumn{4}{c}{$\Gamma_{b}$ (units of $g$ ) } & Curvature \\
\cline { 3 - 6 } Substances & $(\mathrm{Hz})$ & $25 \mathrm{~Hz}$ & $50 \mathrm{~Hz}$ & $75 \mathrm{~Hz}$ & $100 \mathrm{~Hz}$ & $10^{-4}$ \\
\hline Water & 72 & 0.60 & 0.52 & 0.29 & 0.72 & 9.3 \\
$300 \mathrm{ppm}$ PEO & 65 & 0.68 & 0.51 & 0.44 & 1.05 & 14.5 \\
$0.4 \mathrm{mM}$ Triton & 55 & 0.64 & 0.28 & 0.52 & 1.35 & 7.6 \\
\hline \hline
\end{tabular}


an acceleration threshold below unity, a minimum frequency $f_{\text {min }}$, and a steep increase of the critical acceleration curve at high frequencies. This behavior is in contrast to that of highly viscous droplets for which the $\Gamma_{b}(f)$ curve increases monotonically above unity [12]. In Ref. [15] it was shown that a system consisting of a droplet and an air film squeezed between the droplet and the bath resonated at a frequency $f_{R}$ that scaled with the capillary frequency $\sqrt{(} \sigma / M)$, where $M$ was the droplet mass. The resonance allowed the droplet to bounce at an acceleration lower than unity and produced the minimum in the threshold acceleration curve. Consequently, the minimum frequency $f_{\min }$ could be directly related to the surface tension, if we assume that the mass $M$ did not differ notably from one case to another (the droplet radii were similar in all cases). Thus, only the surface tension determined the position of the minima. The $f_{\min }$ values were in good agreement with surface tension measurements.

The droplet viscoelasticity affects the threshold value and the local curvature of the bouncing threshold. The theory in Ref. [15] does not account for non-Newtonian behavior. However, several pertinent conclusions can be drawn based on the theoretical predictions for bouncing Newtonian droplets. First, the increase in the threshold has been shown to be related to an increase in the viscosity [15]. Indeed, the polymer solution was the most viscous solution. The deformation of the droplet induces a fluid flow inside the droplet [20]. The shear stress may enhance the effect of the viscosity, especially at low frequencies, because a larger reduced acceleration $\tilde{\Gamma}=A \omega^{2} / g$ was needed to reach the bouncing threshold. Second, the curvature close to the minimum increases with increasing droplet viscosity [15]. This finding was not observed for the polymer solution, where the local curvature near the minimum was larger than for the other two samples. This behavior can only be attributed to the non-Newtonian properties of the droplet.

Two physical considerations from Ref. [15] can explain non-Newtonian effects on the droplet. First, the energy stored in the droplet is used to produce periodic bouncing. More precisely, the bouncing behavior depends on the time the droplet spends in the oblate and in the prolate forms (only the $Y_{2}^{0}$ mode is considered). Consequently, viscoelasticity must be accounted for at this stage. Second, the internal droplet motion significantly affects the film drainage and the resulting lubrication force. This second consideration is debatable. The coupling of the liquid and the air flows at the interface depends on the surface shear viscosity [31]. The polymer (and even the surfactant) most likely affect the surface viscosity and, consequently, the drainage of the air film.

In the next section, only the water droplet and the polymer solution droplet are considered. The Triton solution was used to clearly separate surface tension effects from viscoelastic effects. The threshold measurements allow the two effects to be distinguished and highlight the role played by viscoelasticity. We now compare the behavior of Newtonian and non-Newtonian droplets.

\section{B. Jumping height}

The bouncing droplet trajectories (see, e.g., Fig. 3) were extracted from the images in Figs. 4 and 5. The motion of the droplet center of mass could essentially be represented by a

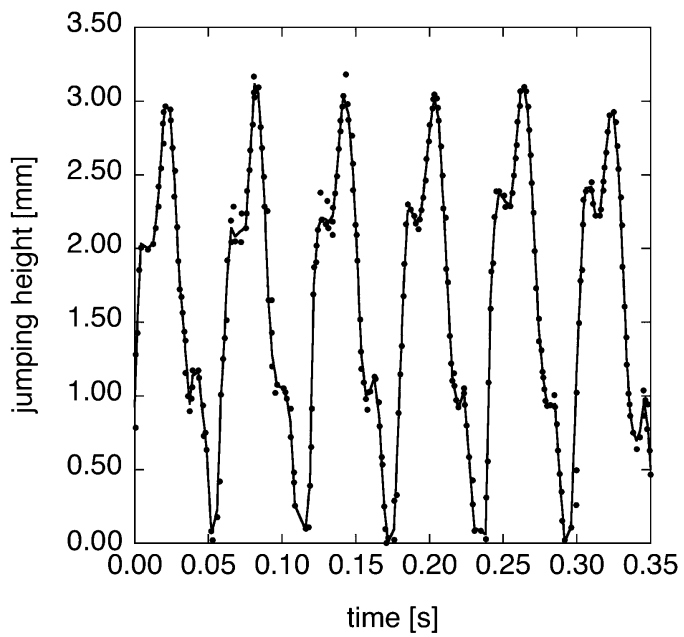

FIG. 3. Periodic trajectory of a bouncing water droplet. The example shown here corresponds to $f=50 \mathrm{~Hz}(\Gamma=4)$. A triply periodic mode is observed.

succession of parabolas. However, period doubling was also observed at large amplitudes, such as $\tilde{\Gamma}=4$. From fitting the experimental data, the heights $s_{\max }$ of the successive maxima were extracted, and the cumulative distribution of heights was plotted (Fig. 6). The values of $s_{\max }$ were determined by analyzing $5 \mathrm{~s}$ movies recorded by the high-speed camera. The regime of interest corresponded to significant droplet deformation, i.e., high acceleration regimes far above the bouncing threshold but still below the Faraday instability. The bouncing behavior was compared for droplets of pure water and the polymer solution. Frequencies (50, 70, and $90 \mathrm{~Hz}$ ) were chosen such that cases below, at, and above $f_{\text {min }}$ of the polymer solution droplets could be studied.

The cumulative distribution exhibited an S-shape, which is typical for a Gaussian distribution. In this case, the mean of the distribution corresponded to $50 \%$ of the cumulative distribution, denoted by $s_{\max , 50}$. When the bouncer maintained a periodic motion, the standard deviation of the distribution was expected to be notably small (see, for example, the classical bouncing ball problem [32,33]). However, the motion was chaotic for a large standard deviation of the distribution.

At $f=50 \mathrm{~Hz}$, the maximum height of the distributions for the water and polymer solution droplets were similar. The indicator $s_{\max , 50}$ was $2.5 \mathrm{~mm}$ for both the water and the aqueous polymer solution droplets, which suggests that the jump height was identical for both cases. Both types of droplets exhibited wide $s_{\max }$ distributions, i.e., the maxima of the trajectories

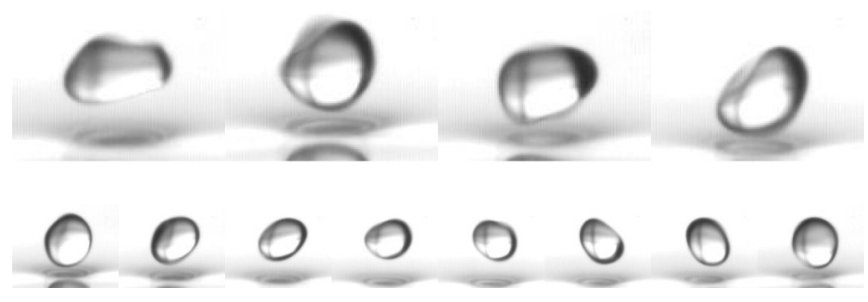

FIG. 4. Top: Example of irregular shape evolution at $90 \mathrm{~Hz}$ for a water droplet. Bottom: Time sequence for a bouncing water droplet at $90 \mathrm{~Hz}$ (time between two images is $1 \mathrm{~ms}$ ). 


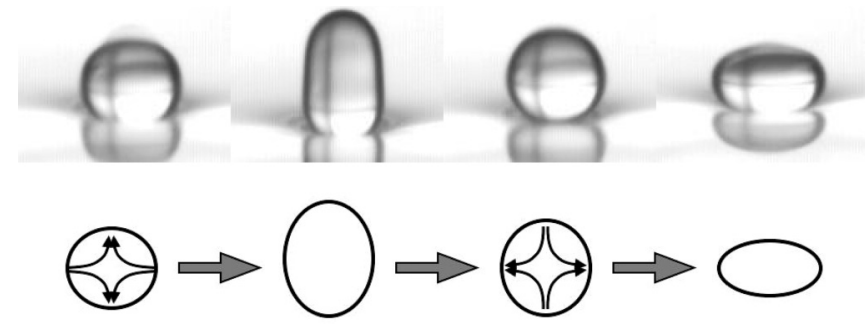

FIG. 5. Top: Typical sequence of shapes for a bouncing droplet of $300 \mathrm{ppm}$ PEO in water at $90 \mathrm{~Hz}$. The droplet shape evolved as follows: sphere, prolate ellipsoid, sphere, and oblate ellipsoid. (The time between two images is $3 \mathrm{~ms}$. The spatial dimensions of the images are $4.5 \times 3.6 \mathrm{~mm}^{2}$ ). Bottom: Sketch of the elongational flow inside a droplet due to deformation during bouncing. A case study of the droplet shape evolution from the oblate state (third picture in top panel) to the spherical state (fourth picture in top panel) is shown.

were nearly uniform between 1 and $4 \mathrm{~mm}$ and exhibited periodic doubling or chaotic motion. Conversely, above the resonance frequency of $f=90 \mathrm{~Hz}$, the distribution rather
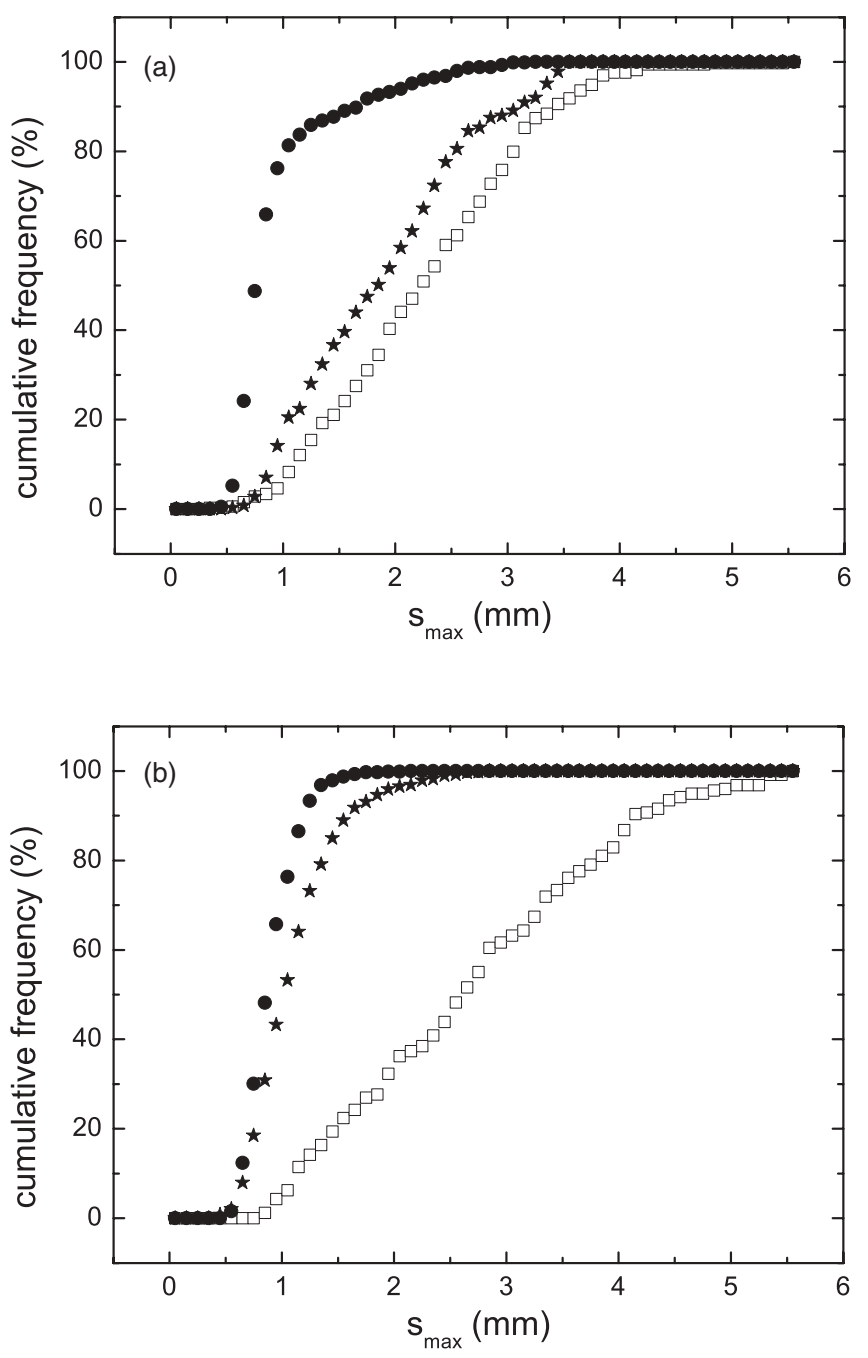

sharply increased around $s_{\max , 50}(0.8 \mathrm{~mm})$ in both cases. This result suggests that the droplets were locked into a periodic mode.

Very different behaviors were observed for the $s_{\max }$ distributions for frequencies near $f_{\min }$, i.e., $f=70 \mathrm{~Hz}$. The $50 \%$ value of $s_{\max }$ was found to be approximately $2.4 \mathrm{~mm}$ for the water droplets and $1 \mathrm{~mm}$ for the polymer solution droplets. The distribution function increases steeply for the polymer solution droplets, whereas the distribution for the pure water droplets was broader, ranging between 1 and $3 \mathrm{~mm}$. The droplet motion of the pure water was chaotic. At the resonance frequency, the non-Newtonian droplet did not bounce as high as the water droplet. This result shows that more energy was dissipated by the internal fluid motion of the polymer solution droplet than for the pure water droplet. In addition, the bouncing heights for the water droplets scaled roughly as $s_{\max , 50} \sim f^{2}$ (data not shown), which was not the case for the polymer solution.

\section{Droplet shape}

To complement the trajectory measurements, droplet deformation was measured at the same frequencies and
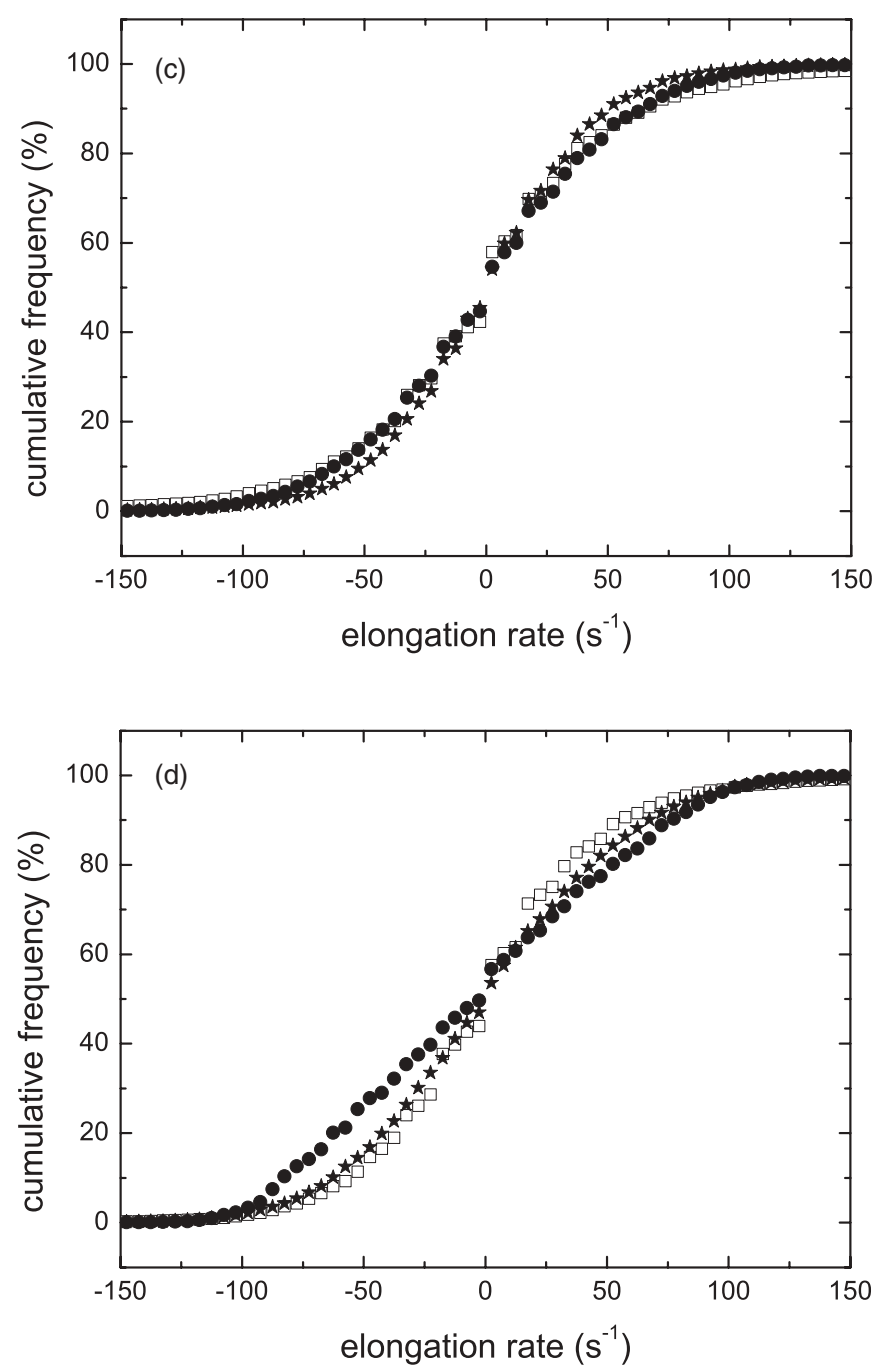

FIG. 6. Cumulative frequency of the maximum jumping height $s_{\max }$ for (a) water droplets and (b) polymer solution droplets at $f=50,70$, and $90 \mathrm{~Hz}$. Cumulative frequency of the elongation rate for (c) water droplets and (d) polymer solution droplets. The accelerations are $\tilde{\Gamma}=4$ at $f=50 \mathrm{~Hz}$ (open squares) and $\tilde{\Gamma}=5$ at $f=70$ (stars) and $90 \mathrm{~Hz}$ (filled circles) for all plots. 
accelerations as used in the trajectory experiments. A bouncing water droplet was once more compared to a droplet consisting of $300 \mathrm{ppm}$ PEO in water to investigate the effect of the polymer on the stability of the droplet shape.

An initial qualitative approach to study the droplet shape was visual examination of the bouncing droplet images. The polymer was found to stabilize the droplets. For the PEO solution at $f=50,70$, and $90 \mathrm{~Hz}$, a highly stable droplet was observed with only minor stretching and compression of the drop shape (Fig. 5).

This regular cycle of elongation and compression was sporadically perturbed during bouncing, but the polymer stabilized the droplet such that the regular shape was recovered within a few milliseconds. The polymer solution droplets showed only slight shape changes. The droplets deformed from a sphere to a stretched ellipsoid, back to a sphere, to a compressed ellipsoid, and finally back to a sphere (see Fig. 5). In this figure, the stabilizing effect of the polymers was most likely caused by the high elongational viscosity of the polymer solutions.

Elongational flow occurs in a bouncing droplet because of the shape change sketched in Fig. 5. Only the flow for the shape change of the droplet from a sphere to a compressed ellipsoid is shown (see the transition from the third to the fourth image in Fig. 5).

Conversely, water droplets bouncing at frequencies of 50 , 70, and $90 \mathrm{~Hz}$ were highly irregular. For these droplets, transitions between the spherical and ellipsoidal (stretched or compressed) limit states could be temporarily observed. When the droplets' regular shape was perturbed, the droplet either did not return to the limit state or returned only briefly to the regular state before the bouncing behavior became irregular again. Figure 4 shows a portion of the deformed droplet shapes at a frequency of $90 \mathrm{~Hz}$.

The deformation caused the droplet to move slightly sideways during bouncing. In most cases, the motion to the left canceled that to the right. The motion was caused by the deformed droplet's failure to hit the surface uniformly, resulting in the droplet rebounding in a direction that was not perpendicular to the horizontal.

In addition to the deformation described above, water droplets were also observed to rotate around their own axis. The superposition of both motions occasionally resulted in droplet tumbling, as shown in Fig. 4. The rotation can be most clearly observed if one follows the motion of the dark edge on the droplet surface (top left in the first image) with respect to time. This deformation caused the droplet to tumble during bouncing.

The deformation was quantified by analyzing the distribution of the droplet elongation rate. When a droplet deforms in a harmonic manner only from a spherical to an oblate shape, the droplet elongation rate can be well approximated from the change in shape during bouncing by using the following equation:

$$
\dot{\varepsilon}=\frac{d \varepsilon}{d t}=-2 \frac{\partial_{t} H(t)}{H(t)},
$$

where $H(t)$ is the vertical height of the polymer solution droplet. $H(t)$ was determined by an edge detection algorithm from LABVIEW (National Instruments, Austin, TX). For the more irregular water droplets, the largest diameter was always chosen, even if the flow differed from an ideal elongational flow in these cases. For the chaotic bouncing water droplets, the direction of maximum elongation and compression changed continuously, and the complete deformation could not described by a single axis of deformation but only by a set of harmonics. However, we are mostly interested in the effect of the polymers on the flow and our analysis provides a good estimate for the elongational rate of the polymeric droplets.

Considering only the length between two subsequent images, the expression above reduces to

$$
\dot{\varepsilon}=-2 \frac{H\left(t_{1}\right)-H\left(t_{2}\right)}{\left(t_{1}-t_{2}\right) H\left(t_{2}\right)},
$$

where $t_{1}$ and $t_{2}$ are the times at which the consecutive images are captured. The cumulative frequency of the occurrence of the elongational rates is given in Fig. 6.

The cumulative frequency for the water droplets did not change significantly. The distribution function increased sharply at at $f=70 \mathrm{~Hz}$ (the resonance frequency), while the distributions at $f=50$ and $90 \mathrm{~Hz}$ were quantitatively identical. The cumulative distributions for the water and polymer solution droplets were nearly superposed at $f=50 \mathrm{~Hz}$ but different at $f=70$ and $90 \mathrm{~Hz}$. Conversely, the cumulative distribution increased monotonically with the frequency for the polymer solution droplets. The distribution of the elongation rates reached the interval $[-100,100] \mathrm{s}^{-1}$ at $f=90 \mathrm{~Hz}$. This result may appear to contradict results showing that polymers increased energy dissipation: however, mostly the higher harmonics in the droplet deformations were suppressed, meaning that the deformation became more regular in the presence of polymers. The droplets were elongated only along one well-defined axis, resulting in a larger droplet elongation and less chaotic motion in this direction. Finally, we should mention that the highest elongational rates in the oscillatory bouncing process were $\dot{\epsilon} \sim 150 \mathrm{~s}^{-1}$, which corresponds to a Weissenberg number of $\mathrm{Wi}=\dot{\epsilon} \lambda \sim 1$.

\section{CONCLUSION}

This work is a comparison between the behaviors of Newtonian and non-Newtonian bouncing droplets. We found that droplet bouncing was affected by a small amount of flexible polymer in the droplet, resulting in an increase in the critical acceleration amplitude. The resonance range for the polymer solution was also shown to be narrower than that for water. Both features may be explained by a theory developed for Newtonian bouncing droplets. The effect of the non-Newtonian fluid on the bouncing mechanism was investigated by analysis and comparison of the trajectory and deformation of droplets during the bouncing process. The nonNewtonian fluid was more effective than water in rendering the bouncing process less chaotic and decreasing the jump height of the droplets, suggesting that more energy was dissipated in the deformation of the polymer solutions droplets than that of the water droplets. This reduction in the chaotic behavior was enhanced at high frequencies. Similar results were obtained from analysis of oscillating elongational droplet deformation. The only material property that can produce such an effect is the high elongational viscosity of the polymer solutions. The 
elongational viscosity of our samples was determined using a CABER setup that measures only the apparent transient elongational viscosity at the deformation rate $\dot{\epsilon}$ defined by the polymer relaxation time. The characteristic relaxation time of the polymer was found to be $\lambda=6.7 \mathrm{~ms}$. Periodic motion was observed for bouncing droplets. The polymer relaxation time $\lambda$ can be used to define a characteristic frequency. The critical acceleration and the regularity of the bouncing process were already affected at the lowest frequency $f=50 \mathrm{~Hz}$. However, the cumulative jumping heights and elongational rates were only marginally affected at a driving frequency of $f=50 \mathrm{~Hz}$, which corresponded to a Deborah number of De $=\lambda_{c} 2 \pi f=2$. While the Weissenberg number compares the polymer relaxation time with the steady shear rate, the Deborah number compares the polymer relaxation time with the characteristic frequency of an oscillatory flow. In principle, the strong effects of elongational flow on polymers should depend on both the Deborah and the Weissenberg numbers, but this dependence is difficult to separate. Numerical simulations may help to elucidate the different dependences. However, we find dependences on both control parameters. The differences in the elongational rates and jumping heights were observable only at stronger driving forces and frequencies above $f=$ $50 \mathrm{~Hz}$. Experimental methods for characterizing the response of low-viscosity polymer solutions to an oscillatory elongation are rare, and the oscillating droplets might present a unique comparison with numerical methods.

\section{ACKNOWLEDGMENTS}

S.D. acknowledges support from FNRS. We thank J. P. Lecomte (Dow Corning, Seneffe, Belgium) for providing silicone oils. This work was also supported by Grants No. COST P21 (physics of droplets) and No. ESA-MAP-Prodex AO-99108 (hydrodynamics of wet foam), and by the DFG Project No. WA1336/4 (droplet detachment of complex liquids).
[1] R. D. Deegan, P. Brunet, and J. Eggers, Nonlinearity 21, C1 (2008).

[2] K. Okumura, F. Chevy, D. Richard, D. Quéré, and C. Clanet, Europhys. Lett. 62, 237 (2003).

[3] A. M. Worthington, The Splash of a Drop (E. \& J. B. Young \& CO, New York, 1895).

[4] A. L. Biance, C. Clanet, and D. Quéré, Phys. Fluids 15, 1632 (2003).

[5] M. Reyssat, J. M. Yeomans, and D. Quéré, Europhys. Lett. 81, 26006 (2008).

[6] M. Rein, Fluid Dyn. Res. 12, 61 (1993).

[7] V. Bergeron, D. Bonn, J. Y. Martin, and L. Vovelle, Nature (London) 405, 772 (2000).

[8] D. Bartolo, A. Boudaoud, G. Narcy, and D. Bonn, Phys. Rev. Lett. 99, 174502 (2007).

[9] T. Gilet, K. Mulleners, J. P. Lecomte, N. Vandewalle, and S. Dorbolo, Phys. Rev. E 75, 036303 (2007).

[10] G. E. Charles and S. G. Mason, J. Colloid Sci. 15, 105 (1960).

[11] X. Chen, S. Mandre, and J. J. Feng, Phys. Fluids 18, 092103 (2006); P. Yue, C. Zhou, and J. J. Feng, ibid. 18, 102102 (2006).

[12] Y. Couder, E. Fort, C.-H. Gautier, and A. Boudaoud, Phys. Rev. Lett. 94, 177801 (2005).

[13] Y. Couder, S. Protière, E. Fort, and A. Boudaoud, Nature (London) 437, 208 (2005).

[14] S. Protière and Y. Couder, Phys. Fluids 18, 091114 (2006).

[15] T. Gilet, D. Terwagne, N. Vandewalle, and S. Dorbolo, Phys. Rev. Lett. 100, 167802 (2008).

[16] T. Gilet, N. Vandewalle, and S. Dorbolo, Phys. Rev. E 76, 035302(R) (2007).
[17] D. Terwagne, N. Vandewalle, and S. Dorbolo, Phys. Rev. E 76, 056311 (2007).

[18] D. Terwagne, T. Gilet, N. Vandewalle, and S. Dorbolo, Phys. Fluids 21, 054103 (2009).

[19] S. I. Lieber, M. C. Hendershott, A. Pattanaporkratana, and J. E. Maclennan, Phys. Rev. E 75, 056308 (2007).

[20] S. Dorbolo, D. Terwagne, N. Vandewalle, and T. Gilet, New J. Phys. 10, 113021 (2008).

[21] J. A. Odell and S. P. Carrington, J. Non-Newtonian Fluid Mech. 137, 110 (2006).

[22] A. Kityk and C. Wagner, Europhys. Lett. 75, 441 (2006).

[23] A. Zell, S. Gier, S. Rafaï, and C. Wagner, J. Non-Newtonian Fluid Mech. 165, 1265 (2010).

[24] F. T. Trouton, Proc. R. Soc. London, A 77, 426 (1906).

[25] P. G. de Gennes, J. Chem. Phys. 60, 5030 (1974).

[26] S. L. Anna and G. H. McKinley, J. Rheol. 45, 115 (2001); L. E. Rodd, T. P. Scott, J. J. Cooper-White, and G. H. McKinley, Appl. Rheol. 15, 12 (2005).

[27] E. Miller, C. Clasen, and J. P. Rothstein, Rheol. Acta 48, 625 (2009).

[28] C. Claasen, Korea-Australia Rheol. J. 60, 331 (2010).

[29] S. Gier and C. Wagner, Phys. Fluids 24, 053102 (2012).

[30] M. Faraday, Philos. Trans. R. Soc. London 121, 299 (1831).

[31] B. Scheid and S. Dorbolo (unpublished).

[32] N. Tufillaro, T. Abbott, and J. Reilly, An Experimental Approach to Nonlinear Dynamics and Chaos (Addison-Wesley, New York, 1992).

[33] T. Gilet, N. Vandewalle, and S. Dorbolo, Phys. Rev. E 79, 055201 (2009). 